\title{
Cytoplasmic CD133 expression correlates with histologic differentiation and is a significant prognostic factor in extrahepatic bile duct cancer and gallbladder cancer
}

\author{
TATSUZO MIZUKAMI $^{1}$, HIROFUMI KAMACHI ${ }^{1}$, TOMOKO MITSUHASHI ${ }^{2}$, TAKAHIRO EINAMA ${ }^{1}$, \\ YUTAKA HATANAKA $^{2}$, TOSHIYA KAMIYAMA ${ }^{1}$ and AKINOBU TAKETOMI $^{1}$ \\ ${ }^{1}$ Department of Gastroenterological Surgery I, Graduate School of Medicine, Hokkaido University, Sapporo, \\ Hokkaido 060-8638; ${ }^{2}$ Department of Surgical Pathology, Hokkaido University Hospital, Sapporo, Hokkaido 060-8648, Japan
}

Received February 26, 2018; Accepted September 13, 2018

DOI: $10.3892 /$ ol.2018.9499

\begin{abstract}
Prominin-1 (CD133) is one of the most important stem cell markers among various malignant tumor types, but the clinicopathological significance of CD133 expression in intrahepatic cholangiocarcinoma remains controversial. To the best of our knowledge, there have been no reports on extrahepatic bile duct cancer (EHBDCA) and gallbladder cancer (GBCA). The present study examined the clinicopathological significance of CD133 expression in EHBDCA and GBCA. Immunohistochemistry was used to evaluate CD133 expression in resected specimens obtained from 82 patients with EHBDCA and GBCA, and this expression was compared with the clinicopathological parameters and survival data of the patients. Cytoplasmic CD133 expression was identified in 20 patients, and its incidence was significantly associated with histopathological grade $(\mathrm{P}=0.035)$, $\mathrm{pT}$ factor $(P=0.020)$ and recurrence $(P=0.046)$. Survival analysis revealed that cytoplasmic CD133 expression in patients was significantly associated with a poorer overall survival (OS) and relapse-free survival (RFS) compared with those without cytoplasmic expression (5-year OS rate, $11.6 \%$ vs. $39.1 \%$; 3 -year RFS rate, $12.5 \%$ vs. $42.0 \%$, respectively). Multivariate analysis revealed that cytoplasmic CD133 expression was an independent prognostic factor for OS and RFS ( $\mathrm{P}=0.0036$
\end{abstract}

Correspondence to: Dr Hirofumi Kamachi, Department of Gastroenterological Surgery I, Graduate School of Medicine, Hokkaido University, North 15, West 7, Kita-ku, Sapporo, Hokkaido 060-8638, Japan

E-mail: hkamachi@db3.so-net.ne.jp

Abbreviations: EHBDCA, extrahepatic bile duct cancer; GBCA, gallbladder cancer; CSCs, cancer stem cells; UICC, Union Internationale Contre le Cancer; DFS, disease-free survival; OS, overall survival

Key words: cluster of differentiation133, cancer stem cell marker, cytoplasmic expression, histological differentiation, extrahepatic bile duct cancer, gallbladder cancer and $\mathrm{P}<0.0001$, respectively). To the best of our knowledge, this is the first report demonstrating that cytoplasmic CD133 expression was associated with histologic differentiation, cancer progression, recurrence and poor prognosis in EHBDCA and GBCA. CD133 expression may be a useful marker for clinical prognosis in patients with EHBDCA and GBCA.

\section{Introduction}

Extrahepatic bile duct cancer (EHBDCA) and gallbladder cancer (GBCA) are rare diseases worldwide, but they are the sixth leading causes of cancer death in Japan, with nearly 17,000 deaths annually (compiled by the Statistics and Information Department, Minister's Secretariat, Ministry of Health, Labour and Welfare). Surgical resection is the only curative treatment, but the 5-year survival rate even after $\mathrm{R} 0$ resection is poor, ranging from 20 to $40 \%$ (1). The incidence of EHBDCA and GBCA is increasing throughout the world with a high fatality rate; therefore, new prognostic markers and treatments for EHBDCA and GBCA patients are required.

Recently, cancer stem cells (CSCs) were reported to play important roles in various kinds of cancer (2). CD133, which is also known as prominin-1, is a cell surface marker of hematopoietic cells and, originally, of progenitor cells. Although the physiological function of CD133 remains to be elucidated, it has been widely used as a marker for CSCs (3-5). Immunohistochemistry has been used to show the clinical significance of CD133 expression in various types of solid tumors including brain tumors (6), breast cancer (7), lung cancer (8), pancreatic cancer (9), hepatocellular carcinoma (10), and colorectal cancer (11), in which positive CD133 expression is significantly associated with poor prognosis. However, the clinicopathological significance of CD133 in intrahepatic cholangiocarcinoma remains controversial (12-14), and there are no reports on EHBDCA and GBCA. Therefore, in this study, we investigated the potential clinical role of CD133 expression, using immunohistochemistry, in a large series of EHBDCA and GBCA patients. 


\section{Patients and methods}

Patient demographics. The participants in this study were 82 patients who underwent radical surgery for EHBDCA and GBCA between 2000 and 2010 at Hokkaido University Hospital, in the Department of Gastroenterological Surgery I, Hokkaido University, Graduate School of Medicine (Sapporo, Japan). The patients' clinicopathological characteristics are summarized in Table I.

The patients' mean age ( \pm standard deviation) was $66.4 \pm 8.7$ years, and 62 patients $(75.6 \%)$ were male and 20 patients $(24.4 \%)$ were female. The predominant sites of the cancer were the proximal bile duct in 32 patients $(39.0 \%)$, distal bile duct in 27 patients (33.0\%), and cystic duct and gallbladder in 23 patients (28.0\%). The pathological T-factor, $\mathrm{N}$-factor, and stage were assigned according to the TNM classification of the Union Internationale Contre le Cancer (UICC). Intraoperative diagnosis of the ductal resection margins was performed using frozen sections. When a positive margin was found, additional resection of the marginal bile duct was performed to the maximum extent possible. R0 curative resection was achieved in 32 patients (39.0\%), and R1 resection was achieved in 50 patients $(61.0 \%)$. The patients' median survival time was 40.7 months.

Recurrence was diagnosed based on clinical examinations and imaging studies. Time to death, final follow-up examination, and the diagnosis of recurrence was measured from the date of surgery. Surviving patients were followed up until May 2014.

Written informed consent was obtained from all 82 patients before enrollment into the study, and this study design and protocol were approved by the institutional Review Board of Hokkaido University Hospital (clinical research approval no. 014-0134).

Pathological specimens. Formalin-fixed and paraffin-embedded specimens were retrieved from the surgical pathology files of the Pathology Department at Hokkaido University Hospital. Sections were cut and stained with hematoxylin and eosin (H\&E) for routine histopathologic examination. All specimens were diagnosed as EHBDCA and GBCA. A representative tissue block was selected from each patient case to perform immunohistochemical studies.

Immunohistochemistry. The resected tissues were fixed in $10 \%$ formalin and embedded in paraffin blocks, and the most representative block was chosen for each patient. Each block was cut into serial $4-\mu \mathrm{m}$-thick sections for staining with $\mathrm{H} \& \mathrm{E}$ and immunohistochemistry for CD133. Immunohistochemistry was performed using the EnVision+ System-HRP (Dako; Agilent Technologies, Inc., Santa Clara, CA, USA).

Briefly, the sections were mounted on charged glass slides, deparaffinized, and rehydrated through a graded ethanol series. Antigens were retrieved in Dako EnVision FLEX Target Retrieval Solution low pH using Dako PT Link for $20 \mathrm{~min}$ at $97^{\circ} \mathrm{C}$, in accordance with the manufacturer's instructions (Dako; Agilent Technologies, Inc.). After blocking endogenous peroxidase activity using $0.03 \%$ hydrogen peroxide, the tissue sections were incubated with a mouse monoclonal antibody
Table I. Clinicopathological characteristics of 82 patients with extrahepatic bile duct cancer and gallbladder cancer.

$\begin{array}{ll}\text { Parameter No. of cases } & \text { Nor }\end{array}$

Age, years (mean \pm standard deviation)

$66.4 \pm 8.7$

Sex

Male/female

Location

$\mathrm{Bp} / \mathrm{Bd} / \mathrm{G}+\mathrm{C}$

Tumor diameter, $\mathrm{cm}$

$<5 / \geq 5$

Histopathological grade

G1, 2/G3

Lymphatic permeation

Negative/positive

Blood vessel permeation

Negative/positive

Perineural invasion

Negative/positive

pT factor

pT1-2/pT3-4

$\mathrm{pN}$ factor

Negative/positive

$36 / 46$

Staging

I, II/III, IV

Resection status

R0/R1

Median survival (months)

Bp, proximal bile duct; Bd, distal bile duct; $\mathrm{G}$, gallbladder; $\mathrm{C}$, cystic duct.

against CD133 (clone W6B3C1 diluted 1:100; Miltenyi Biotec, Tokyo, Japan) at room temperature for $30 \mathrm{~min}$ and then reacted with a dextran polymer reagent combined with secondary antibodies and peroxidase for $30 \mathrm{~min}$ at room temperature. Specific antigen-antibody reactions were visualized with diaminobenzidine chromogen, which was applied for $10 \mathrm{~min}$. Slides were counterstained with hematoxylin, dehydrated, and mounted.

Pancreatic acinar cells were defined as the positive control, and the negative control tissue sections were prepared by omitting the primary antibody.

Immunohistochemical evaluation. All assessments were performed on the tumor region in the whole section (x200). Each slide was evaluated independently by two observers (TaM and ToM) who did not know the clinical outcomes. CD133 expression was defined as positive if any cells stained in the cytoplasm were observed in the tumor, in accordance with previous reports $(10,12)$.

Statistical analysis. We used the Chi-squared or Fisher's exact test toevaluate the relationships between clinicopathologicaldata 
A

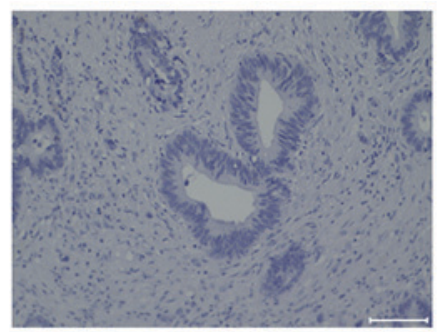

D

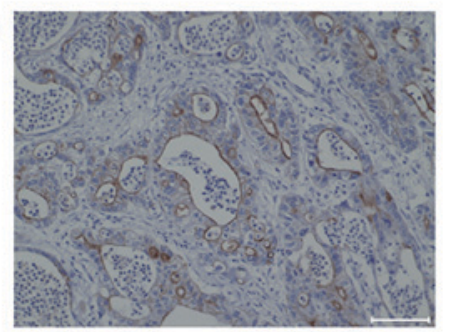

B

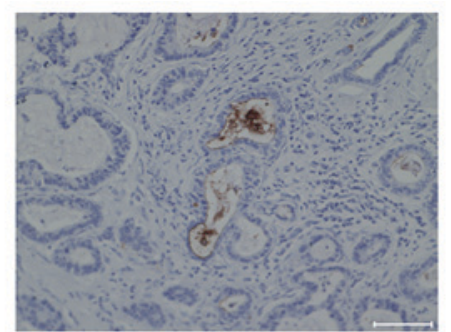

$\mathbf{E}$

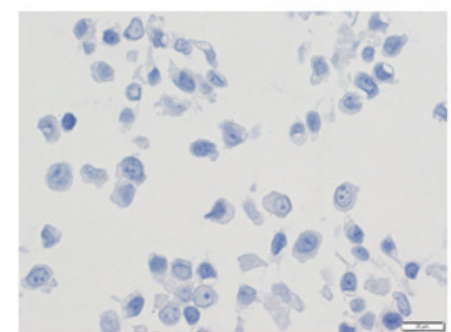

C

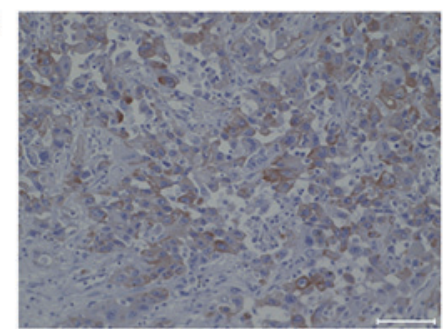

$\mathbf{F}$

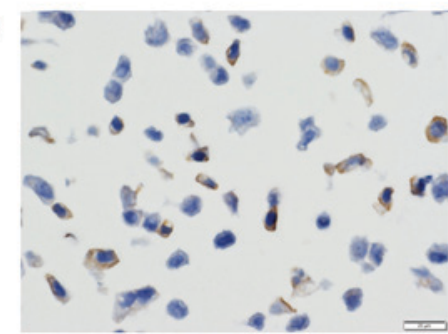

Figure 1. Representative immunohistochemical staining of CD133 in EHBDCA and GBCA cells. (A) No stain for CD133 in tumor cells. (B) Luminal surface and intraluminal cell stain for CD133 in tumor cells. (C) Cytoplasmic stain for CD133 in tumor cells. (D) Mixed stain for CD133 in tumor cells. Scale bar, $100 \mu \mathrm{m}$. (E) MCF-7 cells were used as a negative control and (F) MDA-MB-468 cells were used as a positive control. Scale bar, $20 \mu \mathrm{m}$. CD133, prominin-1; EHBDCA, extrahepatic bile duct cancer; GBCA, gallbladder cancer.

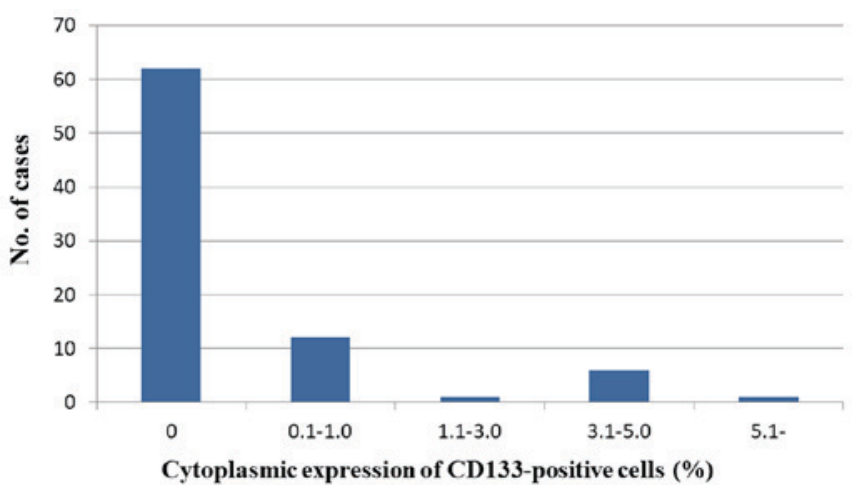

Figure 2. Proportion of cytoplasmic expression of CD133-positive cells. Cells with CD133 expression in the cytoplasm were observed in 20 patients, and the proportion of these cells was $<5 \%$, except in one patient. CD133, prominin-1.

and CD133 expression. Overall survival (OS) and relapse-free survival (RFS) rates were calculated using the Kaplan-Meier method, and comparisons between groups were performed using the log-rank test. A Cox proportional hazards model was used for multivariate analysis. $\mathrm{P}<0.05$ was considered to indicate a statistically significant difference. All statistical analyses were performed using JMP Pro 10 (SAS Institute, Inc., Cary, $\mathrm{NC}, \mathrm{USA})$.

\section{Results}

CD133 expression in EHBDCA and GBCA. Representative stained images of EHBDCA and GBCA are shown in Fig. 1.

Proportion of cells with cytoplasmic CD133-positive expression. We found two types of CD133 expression as follows: C-type is the cytoplasmic staining pattern, and M-type is the luminal membranous staining pattern with or without intraluminal stains. Overall, C-type was observed in 20 (24.4\%) patients whereas M-type was observed in 47 (57.3\%) patients, and overlap between the two types was observed in 9 (11.0\%) patients. Among C-type patients, the frequency of cytoplasm-stained cells in the tumor was $<5 \%$ in most cases (Fig. 2).

Correlation between cytoplasmic CD133 expression and clinicopathological features. Statistical analysis revealed that the incidence of CD133-positive expression was correlated with the histopathological grade $(\mathrm{P}=0.035)$, $\mathrm{pT}$ factor $(\mathrm{P}=0.020)$, and recurrence $(\mathrm{P}=0.048)$. However, there was no significant association between CD133 immunoreactivity and tumor diameter, lymphatic permeation, perineural invasion, $\mathrm{pN}$ factor, staging, or resection status (Table II).

Correlation of cytoplasmic CD133 expression with OS andRFS. There was a significant difference in OS between the patients with and without cytoplasmic CD133 expression ( $\mathrm{P}=0.0002)$. Additionally, patients with cytoplasmic CD133 expression showed significantly worse RFS compared with those without cytoplasmic CD133 expression $(\mathrm{P}<0.0001$; Fig. 3).

Correlation of luminal membrane CD133 expression with OS and RFS. There was a significant difference in OS between the patients with and without luminal membrane CD133 expression $(\mathrm{P}=0.014)$. Additionally, patients with luminal membrane CD133 expression tended to show better RFS compared with those without luminal membrane expression ( $\mathrm{P}=0.078$; Fig. 4).

Univariate and multivariate analyses of $O S$. Univariate analysis showed that OS was significantly correlated with histological grade (G3) and CD133-positive expression. Although perineural invasion also tended to be correlated with OS, the difference was not significant $(\mathrm{P}=0.067)$. Multivariate analysis showed that $\mathrm{G} 3(\mathrm{P}=0.018)$, perineural invasion $(\mathrm{P}=0.040)$, and $\mathrm{CD} 133$-positive expression $(\mathrm{P}=0.0036)$ were independent prognostic factors for OS (Table III). 
Table II. Association between cytoplasmic CD133 expression and clinicopathological features.

Cytoplasmic expression of CD133

Clinicopathological feature

\begin{tabular}{lcl}
\hline Positive $(n=20)$ & Negative $(n=62)$ & P-value \\
$16 / 4$ & $54 / 8$ & 0.474 \\
$11 / 9$ & $49 / 13$ & $0.035^{\mathrm{a}}$ \\
$8 / 12$ & $24 / 38$ & 0.918 \\
$10 / 10$ & $23 / 39$ & 0.306 \\
$3 / 17$ & $14 / 48$ & 0.545 \\
$7 / 13$ & $40 / 22$ & $0.020^{\mathrm{a}}$ \\
$6 / 14$ & $30 / 32$ & 0.150 \\
$8 / 12$ & $36 / 26$ & 0.159 \\
$4 / 16$ & $28 / 34$ & 0.065 \\
$2 / 18$ & $22 / 40$ & $0.046^{\mathrm{a}}$
\end{tabular}

Tumor diameter, $\mathrm{cm}:<5 / \geq 5$

Histopathological grade: G1, 2/G3

Lymphatic permeation: Negative/positive

$0.046^{\mathrm{a}}$

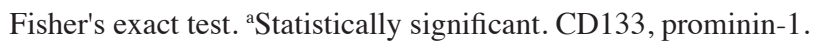
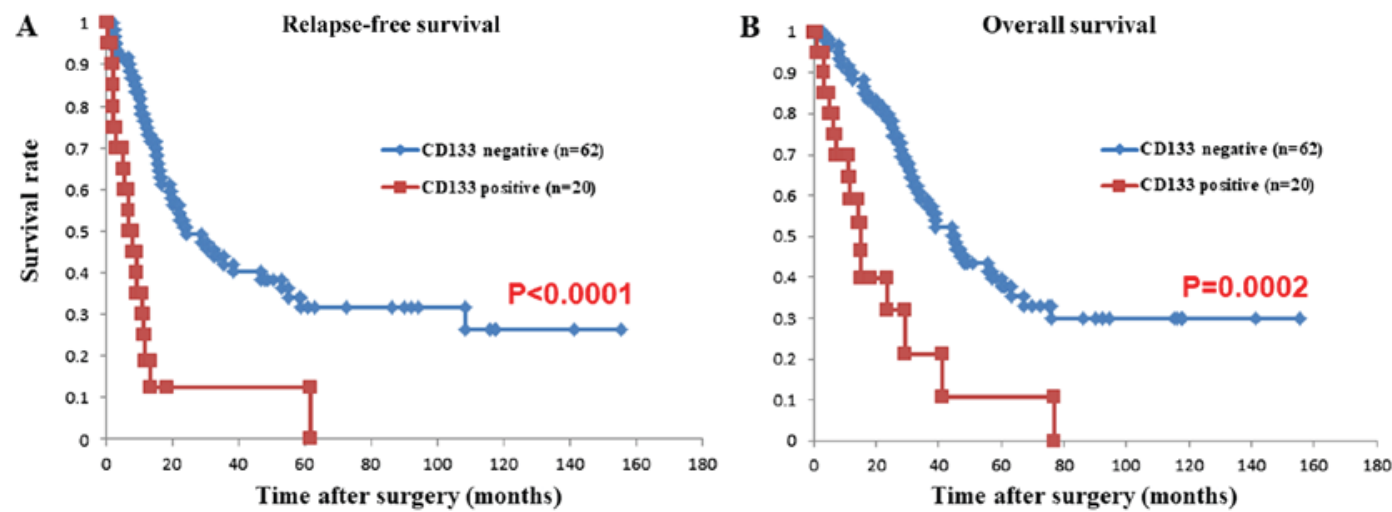

Figure 3. Cytoplasmic expression of CD133 correlates with poor prognosis in EHBDCA and GBCA patients. (A) RFS of EHBDCA and GBCA patients, stratified by their CD133 expression status. The RFS of patients with cytoplasmic CD133 expression was significantly worse than that of the patients without cytoplasmic CD133 expression (3-year DFS rate 12.5\% for those showing cytoplasmic expression vs. $42.0 \%$ for those not showing cytoplasmic expression; $\mathrm{P}<0.0001$ ). (B) OS of EHBDCA and GBCA patients, stratified by their CD133 expression status. The OS of the patients with cytoplasmic CD133 expression was significantly worse than that of the patients without cytoplasmic expression (5-year OS $11.6 \%$ for those showing cytoplasmic expression vs. $39.6 \%$ for those not showing cytoplasmic expression; $\mathrm{P}=0.0002$ ). CD133, prominin-1; EHBDCA, extrahepatic bile duct cancer; GBCA, gallbladder cancer; RFS, relapse-free survival; DFS, disease-free survival; OS, overall survival.
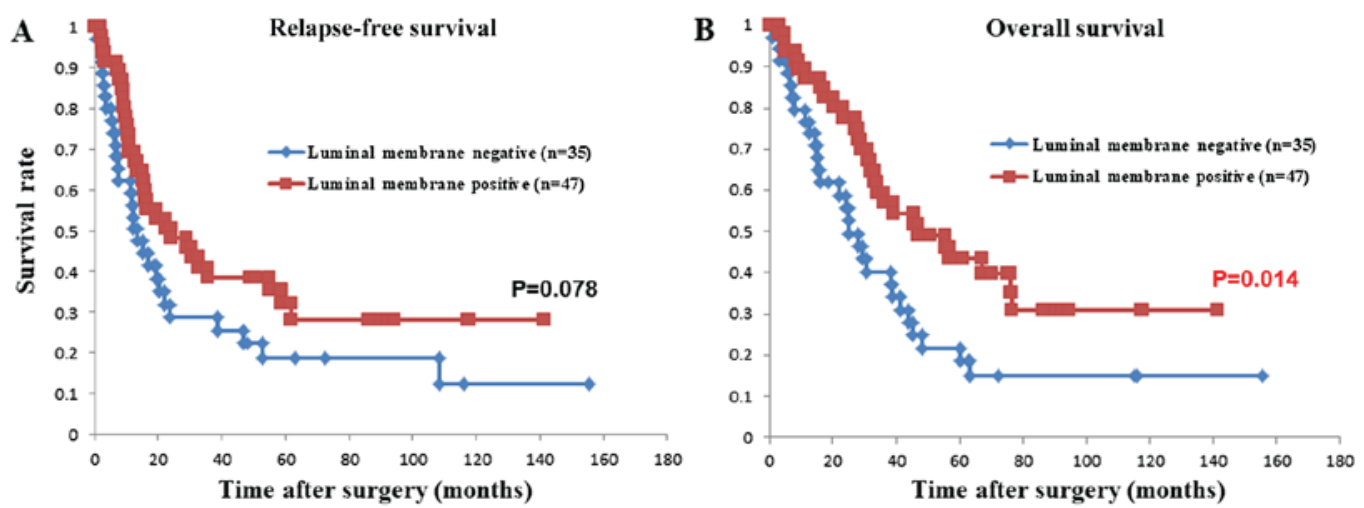

Figure 4. Luminal membrane CD133 expression correlates with a good prognosis in EHBDCA and GBCA patients. (A) RFS of EHBDCA and GBCA patients, stratified by their luminal membrane CD133 expression status. The RFS of patients with positive CD133 expression was better than that of patients with negative CD133 expression, but the difference was not significant (3-year DFS rate 38.4\% for those showing positive CD133 expression vs. 28.7\% for those showing negative CD133 expression; $\mathrm{P}=0.078$ ). (B) OS of EHBDCA and GBCA patients, stratified by their luminal membrane CD133 expression status. The OS of the patients with positive CD133 expression was significantly better than that of the patients with negative CD133 expression (5-year OS $43.3 \%$ for those showing positive $\mathrm{CD} 133$ expression vs. $21.6 \%$ for those showing negative $\mathrm{CD} 133$ expression; $\mathrm{P}=0.014)$. CD133, prominin-1; EHBDCA, extrahepatic bile duct cancer; GBCA, gallbladder cancer; RFS, relapse-free survival; DFS, disease-free survival; OS, overall survival. 
Table III. Univariate and multivariate analyses of prognostic parameters for overall survival in extrahepatic bile duct cancer and gallbladder cancer.

\begin{tabular}{llllll}
\hline & \multicolumn{3}{c}{ Univariate analysis } & & Multivariate analysis \\
\cline { 2 - 3 } Parameters & No. of patients & 5 -year survival rate (\%) & P-value & Hazard ratio (95\% CI) $\quad$ P-value \\
\hline
\end{tabular}

Tumor diameter, $\mathrm{cm}$

$<5$

$\geq 5$

Histopathological grade

G1,

G3

Lymphatic permeation

Negative

Positive

Blood vessel permeation

Negative

Positive

Perineural invasion

$\begin{array}{ll}\text { Negative } & 17 \\ \text { Positive } & 65 \\ \text { pT factor } & \end{array}$

$$
\text { pT1-2 }
$$

pT3-4

$\mathrm{pN}$ factor

Negative

Positive

Staging

I, II

III, IV

Resection status

R0

R1

Cytoplasmic expression of CD133
Negative
Positive

70

12

$$
60
$$

22

32

50

\section{3}

49

\section{7}

65

\section{7}

35

\section{6}

46

\section{4}

38

32

50

20

62
35.5

22.9

40.4

12.4

44.5

26.6

46.0

25.0

56.7

27.2

40.4

22.3

45.8

22.4

39.8

25.6

0.215

$0.001^{\mathrm{b}}$

0.214

0.303

$0.067^{\mathrm{a}}$

0.160

0.142

0.738

0.167

41.3

28.9

39.1

11.6

$<0.001^{\text {b }}$
ND

1

$2.227(1.152-4.122)$

ND

ND

1

$2.101(1.031-4.879)$

ND

ND

ND

ND

$1^{\mathrm{b}}$

$2.947(1.447-5.718)$ $0.018^{\mathrm{b}}$

${ }^{a}$ Factors with $\mathrm{P}<0.100$ were selected for further investigation using a multivariate analysis. ${ }^{\mathrm{b}} \mathrm{A}$ statistically significant result. CD133, prominin-1; CI, confidence interval; ND, not determined.

Univariate and multivariate analyses of RFS. Univariate analysis showed that RFS was significantly correlated with G3, perineural invasion, and CD133 positive expression. Although $\mathrm{pN}$ factor and staging also tended to be correlated with RFS, the difference was not significant $(\mathrm{P}=0.063$ and $\mathrm{P}=0.079$, respectively). Multivariate analysis showed that perineural invasion $(\mathrm{P}=0.045)$ and $\mathrm{CD} 133$-positive expression $(\mathrm{P}<0.0001)$ were independent prognostic factors for RFS (Table IV).

\section{Discussion}

In our study, we used immunohistochemistry to demonstrate the clinical role of CD133 expression in EHBDCA and GBCA.
Our major findings are as follows: (1) Cytoplasmic CD133 expression was closely correlated with poor prognosis and was an independent prognostic factor; (2) cytoplasmic CD133 expression was related to histological differentiation, which is also an independent prognostic factor in EHBDCA and GBCA. There have been three reports to date on the prognostic value of CD133 in intrahepatic cholangiocarcinoma, but the prognostic significance of CD133 remains controversial (12-14). Shimada et al (12) maintained that cytoplasmic CD133 expression was independently related to a worse prognosis, but Leelawat et al (14) demonstrated that, although CD133 expression significantly correlates with lymph node metastasis and surgical margin status, it does not correlate with poor prognosis. Moreover, Fan et al (13) stressed that CD133 expression 
Table IV. Univariate and multivariate analyses of prognostic parameters for recurrence-free survival in extrahepatic bile duct cancer and gallbladder cancer.

\begin{tabular}{|c|c|c|c|c|c|}
\hline \multirow[b]{2}{*}{ Parameters } & \multicolumn{3}{|c|}{ Univariate analysis } & \multicolumn{2}{|c|}{ Multivariate analysis } \\
\hline & No. of patients & $\begin{array}{c}5 \text {-year } \\
\text { survival rate (\%) }\end{array}$ & P-value & $\begin{array}{c}\text { Hazard ratio } \\
\text { (95\% confidence interval) }\end{array}$ & P-value \\
\hline \multicolumn{6}{|c|}{ Tumor diameter, $\mathrm{cm}$} \\
\hline$<5$ & 70 & 36.6 & 0.320 & ND & \\
\hline$\geq 5$ & 12 & 22.9 & & & \\
\hline \multicolumn{6}{|c|}{ Histopathological grade } \\
\hline $\mathrm{G} 1,2$ & 60 & 41.5 & $0.005^{\mathrm{b}}$ & 1 & 0.143 \\
\hline G3 & 22 & 12.0 & & $1.624(0.843-2.992)$ & \\
\hline \multicolumn{6}{|c|}{ Lymphatic permeation } \\
\hline Negative & 2 & 40.6 & 0.202 & ND & \\
\hline Positive & 50 & 29.8 & & & \\
\hline \multicolumn{6}{|c|}{ Blood vessel permeation } \\
\hline Negative & 33 & 44.6 & 0.274 & ND & \\
\hline Positive & 49 & 27.2 & & & \\
\hline \multicolumn{6}{|c|}{ Perineural invasion } \\
\hline Negative & 17 & 52.9 & 0.036 & 1 & $0.045^{\mathrm{b}}$ \\
\hline Positive & 65 & 28.8 & & $2.132(1.016-4.941)$ & \\
\hline \multicolumn{6}{|l|}{ pT factor } \\
\hline pT1-2 & 47 & 38.7 & 0.216 & ND & \\
\hline pT3-4 & 35 & 27.8 & & & \\
\hline \multicolumn{6}{|l|}{$\mathrm{pN}$ factor } \\
\hline Negative & 36 & 44.5 & $0.063^{\mathrm{a}}$ & 1 & 0.896 \\
\hline Positive & 46 & 26.0 & & $1.043(0.559-1.978)$ & \\
\hline \multicolumn{6}{|l|}{ Staging } \\
\hline $\mathrm{I}, \mathrm{II}$ & 44 & 42.2 & $0.079^{\mathrm{a}}$ & 1 & 0.191 \\
\hline III, IV & 38 & 25.0 & & $1.484(0.821-2.700)$ & \\
\hline \multicolumn{6}{|c|}{ Resection status } \\
\hline R0 & 32 & 41.5 & 0.135 & ND & \\
\hline $\mathrm{R} 1$ & 50 & 29.4 & & & \\
\hline \multicolumn{6}{|c|}{ Cytoplasmic expression of CD133 } \\
\hline Negative & 20 & 42.0 & $<0.001^{\mathrm{b}}$ & 1 & $<0.001^{\mathrm{b}}$ \\
\hline Positive & 62 & 12.5 & & $2.947(1.447-5.718)$ & \\
\hline
\end{tabular}

${ }^{\text {a}}$ Factors with $\mathrm{P}<0.100$ were selected for further investigation using a multivariate analysis. ${ }^{\mathrm{b}} \mathrm{A}$ statistically significant result. CD133, prominin-1; CI, confidence interval; ND, not determined.

was correlated with prolonged patient survival. These results seem to be contradictory.

One of the reasons why these results, including ours, are different from each other despite the same type of cancer may be because of the antibodies used. In our study, we selected the clone W6B3C1 from Miltenyi Biotec because this clone is difficult to stain nonspecifically (15), so we suggest that we can obtain more accurate information about site of CD133 expression using the W6B3C1 clone. To assess CD133 expression using immunohistochemistry, we isolated breast cancer cell lines (MCF-7 and MDA-MB-468) using fluorescence activated cell sorting (FACS), as previously reported by Croker et al (16), We confirmed that MCF-7 cells did not show CD133 expression, but MDA-MB-468 cells did consistently express CD133 only on their surface using this clone (Fig. 1).

The opposing results may also be explained by confusing the cytoplasmic expression and luminal membrane CD133 expression. Leelawat et al (14) and Fan et al (13) defined CD133 positivity based on the percentage of stained cells without distinguishing between the staining sites in their papers. Based on a similar definition, CD133 expression on the luminal membrane with or without intraluminal contents resulted in better OS compared with no CD133 expression in our study (Fig. 4).

These results suggested that cytoplasmic, not luminal membrane, CD133 expression may be an important risk factor 
in EHBDCA and GBCA, which is similar to a report by Shimada et al (12).

Several studies also showed a significant correlation between cytoplasmic CD133 expression and poor prognosis in gastric cancer (17), colorectal cancer (11), hepatocellular carcinoma (10), and ovarian cancer (18) patients. Among these papers, some authors suggested that cells with CD133 expression in the cytoplasm may have CSC-like characteristics. They suggested that cytoplasmic CD133 expression is found in only a small population of cells. To be more precise, the percentage of CD133-positive cells was $16.2 \%$ in hepatocellular carcinoma, $48.3 \%$ in cholangiocarcinoma, and $31.2 \%$ in ovarian cancer, but the proportion of the cells with CD133 expression in the cytoplasm was $<10 \%$. These results are in agreement with our data (Fig. 2).

Cytoplasmic CD133 expression is likely to be a powerful prognostic factor and it may have CSCs-like characteristics, but localization of the CD133 antigen in the cytoplasm remains unclear, even though its expression was originally on the cell membrane.

What is the significance of cell membrane and cytoplasmic CD133 expression? The significance of cell membrane CD133 expression was described previously (19). It was reported that CD133 was exclusively expressed on the luminal membrane of gland-forming cells and it was never found on poorly differentiated diffuse-type cells in human gastric cancers. Considering that poorly differentiated tumors often develop from well-differentiated tumors during tumor progression and they are detected using FACS analysis, it was suggested that loss of CD133 expression on the luminal membrane may be related to tumor progression.

However, for the significance of cytoplasmic CD133 expression, Bauer et al revealed the process of differentiation involved in CD133 in hematopoietic stem cells (20). In their report, they explained that during the process of differentiation, hematopoietic progenitors release small membrane vesicles containing the CD133 protein by exportation via exosomes and upon release, CD133 protein-containing membrane vesicles are internalized by feeder cells. It is widely known that some types of proteins gain biological function based on their site of expression. CD133 may be one of these types of proteins and we speculated that a shift from membranous localization to cytoplasmic localization reflects the transition of epithelial cells to a more invasive phenotype, even in EHBDCA and GBCA.

This hypothesis may be supported by the significant association between cytoplasmic CD133 expression and histologic differentiation, and because CD133 is an independent prognostic factor in our study. Thus, understanding its mechanism might allow us to manipulate the fate of these cells for novel molecular target therapies.

In conclusion, we first demonstrated that cytoplasmic CD133 expression was correlated with histologic differentiation and was a significant prognostic factor in EHBDCA and GBCA. Our study suggests that cytoplasmic CD133 expression might be a useful marker for the clinical prognosis of EHBDCA and GBCA patients and that CD133 might be a powerful molecular target for cancer treatment. However, our results were obtained using a relatively small cohort $(n=82)$ of EHBDCA and GBCA patients, and additional studies in larger cohorts are required to clarify the predictive significance.

\section{Acknowledgements}

The authors would like to thank Mrs. Y. Hirano, Mr. T. Shimizu and Mr. K. Marukawa for their technical assistance. The authors would like to thank Dr Jodi Smith for editing a draft of this manuscript.

\section{Funding}

The present study was supported in part by a grant-in-aid from the foundation for the Department of Gastroenterological Surgery I, Hokkaido University Alumni Association.

\section{Availability of data and materials}

The datasets used and analyzed during the current study are available from the corresponding author on reasonable request.

\section{Authors' contributions}

TaM performed the immunohistochemistry, evaluated the immunostaining, performed the statistical analysis and drafted the manuscript. HK designed the study, analyzed the data and helped to revise the manuscript. ToM confirmed the diagnosis of the samples, evaluated the immunostaining and helped to revise the manuscript. TE participated in the follow-up study. YH performed immunohistochemistry and participated in designing the study. TK participated designing the study and analyzed the data. AT supervised the research, analyzed the data and edited the manuscript. All authors read and approved the final manuscript.

\section{Ethics approval and consent to participate}

Ethics approval was requested and obtained from the Institutional Review Board of Hokkaido University Hospital (clinical research approval no. 014-0134). Written informed consent was obtained from all participants.

\section{Patient consent for publication}

Written informed consent was obtained from all participants.

\section{Competing interests}

The authors declare that they have no competing interests.

\section{References}

1. Ruzzenente A, Iacono C, Conci S, Bertuzzo F, Salvagno G, Ruzzenente O, Campagnaro T, Valdegamberi A, Pachera S, Bagante $\mathrm{F}$ and Guglielmi A: A novel serum marker for biliary tract cancer: Diagnostic and prognostic values of quantitative evaluation of serum mucin 5AC (MUC5AC). Surgery 155: 633-639, 2014.

2. Visvader JE and Lindeman GJ: Cancer stem cells in solid tumours: Accumulating evidence and unresolved questions. Nat Rev Cancer 8: 755-768, 2008. 
3. Wu Y and Wu PY: CD133 as a marker for cancer stem cells: Progresses and concerns. Stem Cells Dev 18: 1127-1134, 2009.

4. Irollo E and Pirozzi G: CD133: To be or not to be, is this the real question? Am J Transl Res 5: 563-581, 2013.

5. Li Z: CD133: A stem cell biomarker and beyond. Exp Hematol Oncol 2: 17, 2013.

6. Pallini R, Ricci-Vitiani L, Montano N, Mollinari C, Biffoni M, Cenci T, Pierconti F, Martini M, De Maria R and Larocca LM: Expression of the stem cell marker CD133 in recurrent glioblastoma and its value for prognosis. Cancer 117: 162-174, 2011

7. Zhao P, Lu Y, Jiang X and Li X: Clinicopathological significance and prognostic value of CD133 expression in triple-negative breast carcinoma. Cancer Sci 102: 1107-1111, 2011.

8. Wu S, Yu L, Wang D, Zhou L, Cheng Z, Chai D, Ma L and Tao Y: Aberrant expression of CD133 in non-small cell lung cancer and its relationship to vasculogenic mimicry. BMC Cancer 12: 535 , 2012.

9. Maeda S, Shinchi H, Kurahara H, Mataki Y, Maemura K, Sato M, Natsugoe S, Aikou T and Takao S: CD133 expression is correlated with lymph node metastasis and vascular endothelial growth factor-C expression in pancreatic cancer. Br J Cancer 98: $1389-1397,2008$

10. Sasaki A, Kamiyama T, Yokoo H, Nakanishi K, Kubota K, Haga H, Matsushita M, Ozaki M, Matsuno Y and Todo S: Cytoplasmic expression of CD133 is an important risk factor for overall survival in hepatocellular carcinoma. Oncol Rep 24: 537-546, 2010.

11. Takahashi S, Kamiyama T, Tomaru U, Ishizu A, Shida T, Osaka M, Sato Y, Saji Y, Ozaki M and Todo S: Frequency and pattern of expression of the stem cell marker CD133 have strong prognostic effect on the surgical outcome of colorectal cancer patients. Oncol Rep 24: 1201-1212, 2010.

12. Shimada M, Sugimoto K, Iwahashi S, Utsunomiya T, Morine Y, Imura S and Ikemoto T: CD133 expression is a potential prognostic indicator in intrahepatic cholangiocarcinoma. J Gastroenterol 45 : 896-902, 2010.
13. Fan L, He F, Liu H, Zhu J, Liu Y, Yin Z, Wang L, Guo Y, Wang Z, Yan Q and Huang G: CD133: A potential indicator for differentiation and prognosis of human cholangiocarcinoma. BMC Cancer 11: 320, 2011.

14. Leelawat K, Thongtawee T, Narong S, Subwongcharoen S and Treepongkaruna SA: Strong expression of CD133 is associated with increased cholangiocarcinoma progression. World J Gastroenterol 17: 1192-1198, 2011.

15. Hermansen SK, Christensen KG, Jensen SS and Kristensen BW: Inconsistent immunohistochemical expression patterns of four different CD133 antibody clones in glioblastoma. J Histochem Cytochem 59: 391-407, 2011.

16. Croker AK, Goodale D, Chu J, Postenka C, Hedley BD, Hess DA and Allan AL: High aldehyde dehydrogenase and expression of cancer stem cell markers selects for breast cancer cells with enhanced malignant and metastatic ability. J Cell Mol Med 13: 2236-2252, 2009.

17. Hashimoto K, Aoyagi K, Isobe T, Kouhuji K and Shirouzu K: Expression of CD133 in the cytoplasm is associated with cancer progression and poor prognosis in gastric cancer. Gastric Cancer 17: 97-106, 2014.

18. Ferrandina G, Martinelli E, Petrillo M, Prisco MG, Zannoni G, Sioletic S and Scambia G: CD133 antigen expression in ovarian cancer. BMC Cancer 9: 221, 2009.

19. Fukamachi H, Shimada S, Ito K, Ito Y and Yuasa Y: CD133 is a marker of gland-forming cells in gastric tumors and Sox17 is involved in its regulation. Cancer Sci 102: 1313-1321, 2011.

20. Bauer N, Wilsch-Bräuninger M, Karbanová J, Fonseca AV Strauss D, Freund D, Thiele C, Huttner WB, Bornhäuser M and Corbeil D: Haematopoietic stem cell differentiation promotes the release of prominin-1/CD133-containing membrane vesicles-a role of the endocytic-exocytic pathway. EMBO Mol Med 3: 398-409, 2011.

c) (i) 1 This work is licensed under a Creative Common Cy Attribution-NonCommercial-NoDerivatives 4.0 International (CC BY-NC-ND 4.0) License. 